\title{
Una especie nueva de helecho del género Pteris (Pteridaceae) endémica de Costa Rica
}

\author{
Alexander Fco. Rojas Alvarado ${ }^{1}$ \& Mónica Palacios Ríos ${ }^{2}$ \\ 1 Jardín Botánico Lankester. Universidad de Costa Rica. Apdo. 1031-7050, Cartago, Costa Rica; afrojasa@yahoo.com \\ 2 Instituto de Ecología. Apdo. \#63 Xalapa, Veracruz, México, 91000; monica@ecologia.edu.mx
}

Recibido 06-X-2003. C Corregido 27-V-2005. Aceptado 15-III-2006.

\begin{abstract}
A new species of fern of the genus Pteris (Filicales: Pteridaceae) endemic to Costa Rica. The new fern species Pteris herrerae A. Rojas \& M. Palacios, endemic to Costa Rica, is described. It differs from $P$. decurrens C. Presl in basal segments reduced to 1/5-1/2 of the next segment (vs. 2/3-3/4), basal pinnae not bifurcated (vs. bifurcated), pinnae apex mucronate (vs. acuminate) and segment apex undulate (vs. dentate). It differs from Pteris consanguinea in the elliptic pinnae (vs. oblong), two segments reduced on the base (vs. lack), segments entire to undulate (vs. dentate), basal pinnae without basiscopic lobes (vs. with basiscopic lobes) and segment apex entire to undulate (vs. dentate). Rev. Biol. Trop. 54 (3): 1061-1066. Epub 2006 Sept. 29.
\end{abstract}

Key words: Pteris, new species, Pteridaceae, ferns, Costa Rica.

El género Pteris (Subfamilia Pteridoideae, Pteridaceae) es un género pantropical con aproximadamente 200 especies y caracterizado por: (1) soro en una comisura marginal que conecta el ápice de las venas, con parafisos filamentosos (algunas veces pocos); (2) Indusio fuertemente diferenciado del margen recurvado; y (3) esporas tetrahédricas o globosas, triletes, usualmente con un ala ecuatorial y pequeños tubérculos más o menos fusionados en el borde, o tubérculos prominentes, o reticuladas. Además presentan distintos tipos de arquitectura laminar y venación variando de libres a areoladas (Prado y Windish 2000).

Varios trabajos recientes han hecho aportes importantes al género con la descripción de especies nuevas, nuevos registros y clarificación de especies, entre ellos: Prado (1993) da un nuevo nombre a $P$. gracilis Fée (nombre ilegítimo) y la nombra como $P$. congesta J. Prado y cambia de estatus a $P$. tristicula Raddi como variedad de $P$. denticulata Sw.; Arbeláez (1995) describe a P. muricatopedata Arbeláez y P. albertiae Arbeláez como especies nuevas y registra dos especies más para Colombia; Lellinger (1997) donde redefine las especies $P$. deflexa y $P$. polita y aclara el uso de otros nombres y; Prado y Smith (2002) describen a P. boliviensis Prado \& A. R. Sm. y P. krameri Prado \& A. R. Sm. y registran una especie más para Bolivia.

Como resultado de la revisión de algunas especies de Pteris de México y Centroamérica, se describe una nueva especie de la familia Pteridaceae de Costa Rica.

\section{MATERIALES Y MÉTODOS}

La nueva especie ha sido así considerada después de revisar las colecciones de diversos herbarios como: Museo Nacional de Costa Rica (CR), Field Museum of Natural History (F), Instituto Nacional de Biodiversidad (INB), Universidad Nacional Autónoma de México (MEXU), Missouri Botanical Garden (MO), 
New York Botanical Garden (NY), y de comparar con especies afines en distintos tratamientos taxonómicos para el Neotrópico tales como (Gómez 1976, 1978, Lellinger 1989, Mickel 1992, Mickel y Beitel 1988, Moran 1995, Proctor 1977, 1985, 1989, Smith 1981, 1995, Stolze 1981, Tryon y Stolze 1989), entre otros.

\section{RESULTADOS}

Pteris herrerae A. Rojas et M. Palacios, sp. nov.

Tipo: COSTA RICA. Limón: Cantón de Talamanca, Cerro Muchilla, Valle de La Estrella, 948' N, 8305' W, 750 msnm, G. Herrera \& E. Madrigal 2599 (Holotipo: CR, 148612; Isotipo: MO). (Fig. 1).

Diagnósis latina: Ab P. consanguinea pinnae eliptica reducto in regione basali, pinnae basalis obsita furcata, segmentis integra vel undulata aberrans.

English diagnosis: The new species differs from $P$. consanguinea by elliptic pinna reduced on the base, basal pinna unfurcate and entire to undulate segments.

Descripción: Terrestres; rizoma no visto (presumiblemente compacto, ascendente); hojas de 1.3-1.7 m de largo, monomórficas, erectas; pecíolo de 2/5-1/2 del tamaño de la hoja, pajizo a pardo-amarillento, liso, glabro; lámina de 50-60 cm de ancho, 1-pinnado-pinnatífida en su totalidad, lanceolada, cartácea, la base truncada; pinnas basales pinnatífidas, equiláteras, sin segmento basiscópico alargado; pinnas suprabasales 6-8 pares, 5-12 cm de ancho, lanceoladas a deltado-lanceoladas, base abruptamente reducida, los segmentos basales reducidos a 1/5-1/2 de los siguientes, las pinnas pediculadas o al menos con la base cuneada, el pedículo hasta $0.5 \mathrm{~cm}$, ápice largamente acuminado a mucronado, el mucron 3-6 cm de largo; pinna terminal igual a las pinnas laterales; últimos segmentos rectos a levemente curvados; raquis y costas glabros, pajizos, inermes, aristados adaxialmente, las aristas de menos de $0.5 \mathrm{~mm}$, firmes; nervadura parcialmente areolada, con una serie de areolas sobre las costas y cóstulas; soros cubriendo la mayor parte del margen de los segmentos, con el ápice libre y a veces una pequeña parte del seno.

Distribución: Conocida sólo en la vertiente caribe de la Cordillera de Talamanca a 750 msnm Costa Rica y probablemente Panamá. Paratipo: COSTA RICA. Cartago: Río Chitarria, E of Buena Vista, NE $20 \mathrm{~km}$ from Turrialba, 750 msnm Y. Saiki CR-63 (F).

Etimología: Esta especie es dedicada a Gerardo Herrera, quien por mucho tiempo ha realizado colectas muy importantes de plantas en el Costa Rica.

\section{DISCUSIÓN}

Se parece a Pteris consanguinea Mett. ex Kuhn (Cuadro 1) de Venezuela por tener la lámina 1-pinnado-pinnatífida y nervadura reticulada, pero difiere por sus pinnas lanceoladas a deltado-lanceoladas (vs. oblongas) con un par de segmentos reducidos en la base (vs. sin ellos), pinnas basales no bifurcadas (vs. bifurcadas) y segmentos enteros a ondulados (vs. dentados).

Se parece también a $P$. decurrens C. Presl (Cuadro 1) de Colombia, Ecuador, Perú, Bolivia, Venezuela, Brasil y Chile por tener la lámina 1-pinnado-pinnatífida y nervadura parcialmente areolada, pero los segmentos basales se reducen a 1/5-1/2 del tamaño de los segmentos siguientes (vs. 2/3-3/4), pinnas basales no bifurcadas (vs. bifurcadas), ápice de las pinnas mucronado (vs. acuminado) y ápice de los segmentos ondulado (vs. dentado).

Por la forma de la lámina y los segmentos basales de las pinnas reducidos se parece a $P$. paucinervata Fée (Cuadro 1) del S. México y Mesoamérica, pero se puede diferenciar por el pecíolo pajizo a pardo-amarillento (vs. pardo oscuro a atropurpúreo); pinnas basales equiláteras, sin pínnula basiscópica basal desarrollada 


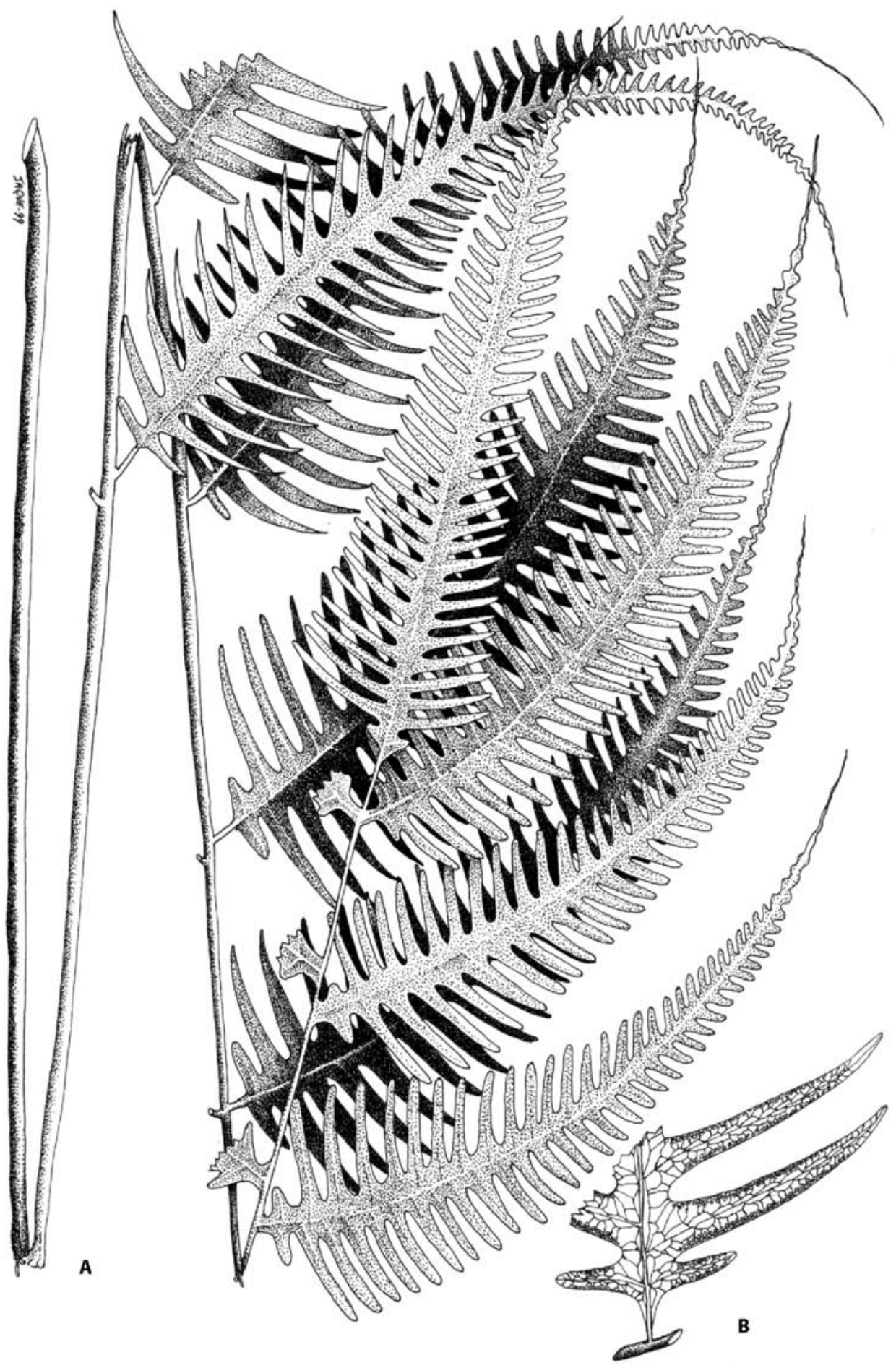

Fig. 1. Pteris herrerae (G. Herrera \& E. Madrigal 2599, CR). A. Hoja. B. Base de pinna media.

Fig. 1. Pteris herrerae (G. Herrera \& E. Madrigal 2599, CR). A. Leaf. B. Base of middle pinna. 
CUADRO 1

Diferencias morfológicas entre P. herrerae y algunas especies emparentadas (Pteris consanguinea, P. decurrens $y$ P. paucinervata).

TABLE 1

Morphological difference between P. herrerae and some related species (Pteris consanguinea, P. decurrens and P. paucinervata)

P. herrerae

\begin{tabular}{|c|c|}
\hline Tamaño de la hoja & 1.3-1.7 m de largo \\
\hline Color del pecíolo & $\begin{array}{l}\text { pajizo a pardo- } \\
\text { amarillento }\end{array}$ \\
\hline Pinnas basales & no bifurcadas \\
\hline $\begin{array}{l}\text { Pinnas supra- } \\
\text { basales }\end{array}$ & 6-8 pares \\
\hline Forma de las pinnas & $\begin{array}{l}\text { lanceoladas a } \\
\text { deltado-lanceoladas }\end{array}$ \\
\hline Ápice de las pinnas & mucronado \\
\hline Segmentos basales & $\begin{array}{l}\text { reducidos a } 1 / 5-1 / 2 \\
\text { del tamaño de los } \\
\text { segmentos siguientes }\end{array}$ \\
\hline Segmentos & $\begin{array}{l}\text { con la base y región } \\
\text { media entera y ápice } \\
\text { ondulado }\end{array}$ \\
\hline Nervadura & areolada \\
\hline Número de areolas & $\begin{array}{l}\text { una serie entre la } \\
\text { cóstula y el margen }\end{array}$ \\
\hline
\end{tabular}

P. decurrens

0.6-2 m de largo

pardo claro a pardo oscuro

bifurcadas

3-6 pares

oblongo-lanceoladas a

elípticas

acuminado a

cuspidado

reducidos a 2/3-3/4

del tamaño de los

segmentos siguientes

con la base y región
media entera y ápice
dentado

areolada

dos series entre la cóstula y el margen
P. consanguinea

P. paucinervata

0.6-1.2 m de largo

0.6-1.3 m de largo

pardo-amarillento

pardo oscuro a atropurpúreo

bifurcadas

bifurcadas

5-8 pares

3-7 pares

oblongas

lanceoladas

acuminado a

cuspidado

no reducidos

reducidos a 2/3-3/4 del tamaño de los segmentos siguientes
con la base y región media ondulada a dentada y ápice dentado

con la base y región media entera y ápice ondulado

areolada

libre

dos series entre la cóstula y el margen no aplica (vs. pinnas basales inequiléteras con pínnula basiscópica basal 1.5-3 veces más largas que las restantes); pinnas y segmentos perpendiculares o casi así (vs. pinnas y segmentos ascendentes); nervadura reticulada (vs. libre) y pinnas con ápice mucronado (vs. agudo).

Por la lámina 1-pinnado-pinnatífida y pinnas basalmente reducidas con el ápice mucronado se parece a Pteris pungens Willd. de México hasta Panamá, Las Antillas, Colombia, Venezuela, Guayanas, Ecuador,
Perú y Bolivia, pero se diferencia por hojas de 130-160 cm de largo (vs. 30-130 cm); pinnas basales equiláteras, sin pínnula basiscópica basal desarrollada (vs. pinnas basales inequiléteras con pínnula basiscópica basal 2-3 veces más largas que las restantes); pinnas suprabasales 5-7 pares (vs. 1-5 pares); base de las pinnas abruptamente reducida (vs. gradualmente reducida); nervadura reticulada, con una serie de areolas paralelas a las costas y a las cóstulas (vs. nervadura libre). 
En Mesoamérica $P$. speciosa Mett. ex Kuhn de Costa Rica, Panamá, Colombia, Ecuador y Perú es la única especie conocida con lámina 1-pinnado pinnatífida y con las pinnas basales equiláteras, pero esta nueva especie puede diferenciarse por el pecíolo y raquis pajizo a pardo-amarillento (vs. pardo oscuro a atropurpúreo), escasamente escamoso en la base (vs. densamente escamoso); pinnas con los segmentos basales reducidos 1/4-1/2 del tamaño de los restantes (vs. segmentos basales del mismo tamaño que los otros), estos no falcados o escasamente así (vs. falcados), el ápice de las pinnas mucronado (vs. agudo); nervaduras en una serie paralela a las costas y las cóstulas con las venas marginales libres (vs. nervaduras en varias series de areolas en las costas y cóstulas).

Arbeláez (1996) utiliza el nombre Pteris horizontalis (Fée) Rosenst. para referirse a $P$. decurrens y en su descripción indica que dicha especie pocas veces tiene las pinnas basales bifurcadas e indica que la lámina es glabra; al contrario, Prado y Windisch (2000) la mencionan con la presencia de una bifurcación basiscópica en las pinnas basales y con tejido laminar peloso. Por un lado, ninguna de las especies más relacionadas ( $P$. consanguinea, $P$. decurrens y $P$. herrerae) tiene la lámina glabra, por lo que puede pensarse que Arbeláez (1996) subestimó los pequeños pelos dispersos de la lámina; por otro, la presencia de pinnas basales bifurcadas o no bifurcadas se puede interpretar como que posiblemente tanto $P$. decurrens como $P$. herrerae están presentes en Colombia, lo cual aún no ha sido corroborado por los autores.

\section{AGRADECIMIENTOS}

La realización de este artículo ha sido posible gracias al convenio INBio-SINAC para el inventariado de las áreas silvestres protegidas y al financiamiento del Gobierno de Holanda. Agradecemos el apoyo de los herbarios por permitirnos utilizar sus colecciones y en algunos casos por el préstamo de material. Enviamos un agradecimiento especial a David
Lellinger y Robbin C. Moran por la revisión del manuscrito y a Francisco Quedada por su excelente ilustración. El segundo autor agradece a Héctor Hernández sus atinadas sugerencias, y el apoyo financiero a Conacyt (4102P-N9607; 35123-V), Conabio y al Instituto de Ecología, A.C. (902-14).

\section{RESUMEN}

Se describe Pteris herrerae A. Rojas \& M. Palacios, endémica de Costa Rica. Esta es diferente de $P$. decurrens C. Presl por segmentos basales reducidos a 1/5-1/2 del tamaño de los siguientes (vs. 2/3-3/4), pinnas basales no bifurcadas (vs. bifurcadas), ápice de las pinnas mucronado (vs. acuminado) y ápice de los segmentos ondulado (vs. dentado). También es diferente de Pteris consanguinea Mett. ex Kuhn por pinnas deltado-lanceoladas (vs. oblongas), con un par de segmentos reducidos en la base (vs. sin ellos), pinnas basales sin lóbulos basicópicos alargados (vs. con lóbulos basiscópicos) y segmentos enteros a ondulados (vs. dentados).

Palabras clave: Pteris, nuevas especies, Pteridaceae, helechos, Costa Rica.

\section{REFERENCIAS}

Arbeláez, A.L. 1995. Two new species and new records for Pteris (Pteridaceae) from Colombia. Brittonia 47: 175-181.

Arbeláez, A.L. 1996. 18. La Tribu Pteridae (Pteridaceae), p. 10-104. In P. Pinto (ed.). Flora de Colombia. Universidad Nacional de Colombia, Bogotá, Colombia.

Gómez, L.D. 1976. Contribuciones a la Pteridología Centroamericana. 1. Enumeratio filicum nicaraguensium. Brenesia 8: 41-57.

Gómez, L.D. 1978. Contribuciones a la Pteridología Centroamericana. 3. Sertum nicaraguense. Brenesia 14-15: 279-281.

Lellinger, D.B. 1997. Pteris deflexa and its Allies. Amer. Fern J. 87: 66-70.

Lellinger, D.B. 1989. The ferns and fern-allies from Costa Rica, Panamá, and The Chocó. Part I. Psilotaceae through Dicksoniaceae. Pteridología 2A: 1-364.

Mickel, J.T. 1992. Pteridophytes, p. 120-431. In R. McVaugh (ed.). Flora Novo-Galiciana. Volume 17. Gymnosperms and Pteridophytes. Michigan, EEUU. 
Mickel, J.T. \& J.M. Beitel. 1988. Pteridophyte Flora of Oaxaca, México. Mem. New York Bot. Gard. 46: 320-327.

Moran, R.C. 1995. Pteris, p. 140-144. In R.C. Moran \& R. Riba (eds.). Flora Mesoamericana. Volumen 1. Psilotaceae a Salviniaceae. Universidad Nacional Autónoma de México, México D.F., México.

Prado, J. 1993. New name and new status in Brazilian Pteris L. (Pteridaceae). Amer. Fern J. 83: 131-134.

Prado, J. \& A.R. Smith. 2002. Novelties in Pteridaceae from South America. Amer. Fern J. 92: 105-111.

Prado, J. \& P.G. Windisch. 2000. The genus Pteris L. (Pteridaceae) in Brazil. Bol. Inst. Bot. 13: 103-199.

Proctor, G.R. 1977. Pteridophyta, p. 1-414. In R.A. Howard. (ed.). Flora of the Lesser Antilles. (Vol. 2). Harvard University, Cambridge, Massachusetts, EEUU.
Proctor, G.R. 1985. Ferns of Jamaica, a guide to the Pteridophytes. British Museum of Natural History, Londres, Inglaterra. 631 p.

Proctor, G.R. 1989. Ferns of the Puerto Rico and The Virgin Islands. Mem. New York Bot. Gard. 53: 1-389.

Smith, A.R. 1981. Pteridophytes, p. 1-370. In D.E. Breedlove (ed.). Flora of Chiapas. Part 2. Calif. Acad. Sci., San Francisco, California, EEUU.

Smith, A.R. 1995. Pteridophytes, p. 1-334. In P.E. Berry, B.K. Holst \& K. Yatskievych (eds.). Flora of the Venezuelan Guayana. Volume 2. Pteridophytes, Spermatophytes, Acanthaceae-Araceae. Timber, Oregón, EEUU.

Stolze, R.G. 1981. Ferns and fern allies of Guatemala. Part II. Polypodiaceae. Fieldiana, Bot, n.s. 6: 446-458.

Tryon, R.M. \& R.G. Stolze. 1989. Pteridophyta of Perú. Part II. 13. Pteridaceae-15. Dennstadtiaceae. Fieldiana, Bot, n.s. 22: 70-81. 